\title{
HYPERPLANES IN THE SPACE OF CONVERGENT SEQUENCES AND PREDUALS OF $\ell_{1}$
}

\author{
E. CASINI, E. MIGLIERINA, AND Ł. PIASECKI
}

\begin{abstract}
The main aim of the present paper is to investigate various structural properties of hyperplanes of $c$, the Banach space of the convergent sequences. In particular, we give an explicit formula for the projection constants and we prove that an hyperplane of $c$ is isometric to the whole space if and only if it is 1-complemented. Moreover, we obtain the classification of those hyperplanes for which their duals are isometric to $\ell_{1}$ and we give a complete description of the preduals of $\ell_{1}$ under the assumption that the standard basis of $\ell_{1}$ is weak*-convergent.
\end{abstract}

\section{INTRODUCTION}

The present paper is mainly devoted to investigate the structural properties of the closed hyperplanes of the Banach space $c$ of the convergent sequences. This study reveals that this class of spaces are very interesting since it provides a complete isometric description of the preduals of the Banach space $\ell_{1}$ when it is assumed that the standard basis of this space is weak*-convergent.

The starting point of our work is a result that lists some properties about the hyperplanes of $c_{0}$. More specifically, the following essentially known theorem summarizes some characterizations of the 1-complemented hyperplanes in $c_{0}$. We prefer to give a short proof of this result for the sake of the convenience of the readers. Indeed, some of the quoted known facts are scattered throughout the literature and a simple remark, based on a well known property of $\ell_{\infty}$, is easy but not immediate.

Theorem 1.1. Let $f \in \ell_{1}$ be such that $\|f\|_{\ell_{1}}=1$. Let us consider the hyperplane $V_{f}=\operatorname{ker} f \subset c_{0}$. The following statements are equivalent

(1) $V_{f}$ is 1-complemented,

(2) $V_{f}^{*}$ is isometric to $\ell_{1}$,

(3) there exists an index $j_{0}$ such that $\left|f_{j_{0}}\right| \geq \frac{1}{2}$,

(4) $V_{f}$ is isometric to $c_{0}$.

Proof. First, we recall that (1) is equivalent to (3) (see [3]) and (1) implies (4) since the 1-complemented infinite dimensional subspaces of $c_{0}$ are isometric to the whole $c_{0}$ (see, e.g., 7]). Trivially, (4) implies (2). Finally, we show that (2) implies (1). By (2) there exists an isometry $T: V_{f}^{*} \rightarrow \ell_{1}$ hence also $T^{*}: \ell_{\infty} \rightarrow V_{f}^{* *}$ is an isometry. By Proposition 5.13, p.142 in [5], there exists a norm-1 projection

$$
P: \ell_{\infty} \rightarrow V_{f}^{* *} \text {. }
$$

Therefore, since $V_{f}^{* *}=[f]^{\perp}=\left\{x^{* *} \in \ell_{\infty}: x^{* *}(f)=0\right\}$, Corollary 2 in [2] implies that $V_{f}$ is 1-complemented in $c_{0}$.

One may ask whether a similar result is true when the space $c_{0}$ is replaced by $c$. Therefore, the main aim of the present paper is to investigate the properties of hyperplanes in $c$. In particular, we would like to determine what the implications

2010 Mathematics Subject Classification. Primary: 46B45. Secondary:46B04.

Key words and phrases. Space of convergent sequences, Projection, $\ell_{1}$ - predual, Hyperplane. 
of Theorem 1.1 are preserved when we consider $c$ instead of $c_{0}$. This study allow us to show that the behavior of hyperplanes in $c$ is much richer than in $c_{0}$. Indeed, we will show that the counterpart in $c$ of Theorem 1.1 is the following result.

Theorem 1.2. Let $f \in \ell_{1}$ be such that $\|f\|_{\ell_{1}}=1$ and let $W_{f}=\operatorname{ker} f \subset c$. Let us consider the following properties:

(1) $W_{f}$ is 1-complemented;

(2) $W_{f}$ is isometric to $c$;

(3) there exists $j_{0} \geq 2$ such that $\left|f_{j_{0}}\right| \geq \frac{1}{2}$;

(4) $W_{f}^{*}$ is isometric to $\ell_{1}$;

(5) there exists $j_{0} \geq 1$ such that $\left|f_{j_{0}}\right| \geq \frac{1}{2}$;

(6) $W_{f}$ is isometric to $c_{0}$;

(7) $\inf _{P}\|P\|=2$ (where $P: c \rightarrow W_{f}$ is a projection);

(8) $\left|f_{1}\right|=1, f_{j}=0$ for every $j \geq 2$.

Then the following implications hold

$$
(1) \Leftrightarrow(2) \Leftrightarrow(3) \Rightarrow(4) \Leftrightarrow(5) \Leftarrow(6) \Leftrightarrow(7) \Leftrightarrow(8) .
$$

The previous theorem is the main result of our paper and in order to prove it we need a number of intermediate results that are interesting in themselves. First of all, we investigate the properties of the projections on the hyperplanes of $c$. Indeed, in Section 2 , by following the approach outlined in 3 . for $c_{0}$, we characterize the 1-complemented hyperplanes of $c$ and we establish a formula to compute the projection constant of a given hyperplane in $c$. The second step (Section 3) is to study the hyperplanes of $c$ that are isometric to $c$ and $c_{0}$ respectively. It is worth to mention that such situations appear only when the projection constant of the hyperplane attains respectively its minimum and maximum. Indeed we show that a hyperplane of $c$ is isometric to $c$ itself if and only if it is 1-complemented. Moreover, the only hyperplane of $c$ that is an isometric copy of $c_{0}$ has projection constant 2 and it is the "natural" one, i.e. the subspace of $c$ whose elements are the vanishing sequences. At the beginning of Section 4 . Proposition 4.1 characterizes the hyperplanes of $c$ whose duals are isometric copies of $\ell_{1}$. The most interesting situations among the class of the spaces $W_{f}$ such that its dual is $\ell_{1}$ occur when $W_{f}$ is isometric neither to $c$ nor to $c_{0}$. Therefore in these particular cases we compute the $\sigma\left(\ell_{1}, W_{f}\right)$-limit of the standard basis of $\ell_{1}$ by explicitly describing the duality between $W_{f}$ and $\ell_{1}$ (see Theorem 4.3). This theorem allow us to obtain two interesting structural results. First, by using a result of [1], we show that a $\ell_{1}$-predual space $X$ is an isometric copy of $W_{f}$ for a suitable choice of the functional $f \in \ell_{1}$, whenever the standard basis of $\ell_{1} \simeq X^{*}$ is assumed to be weak*-convergent. Second, we characterize the hyperplanes $W_{f}$ that are $\ell_{1}$-preduals and are isometric to a quotient of some $C(\alpha)$ where $C(\alpha)$ denotes the space of all continuous realvalued functions on the ordinals less than or equal to $\alpha$ with the order topology.

In the sequel, whenever $X$ is a Banach space, $B_{X}$ denotes the closed unit ball of $X$ and $[x]$ the linear span of a vector $x \in X$. We write $X \simeq Y$ when $X$ and $Y$ are isometrically isomorphic. We also use standard duality between $c$ and $\ell_{1}$, that is, for $x \in c$ and $f \in \ell_{1}: f(x)=\sum_{i=0}^{\infty} f_{i+1} x_{i}$ where $x_{0}=\lim _{i \rightarrow \infty} x_{i}$. Throughout all the paper the hyperplane $W_{f} \subset c$ stands for the kernel of $f \in \ell_{1}$ with $\|f\|_{\ell_{1}}=1$.

\section{The PROJECTIONS ON THE HYPERPLANES OF $c$}

The aim of this section is to extend to $c$ the study of the projections onto the hyperplanes of $c_{0}$ developed in [3]. 
HYPERPLANES IN THE SPACE OF CONVERGENT SEQUENCES AND PREDUALS OF $\ell_{1} 3$

First, the following lemma establishes a formula to compute the norm of a given projection on an hyperplane of $c$.

Lemma 2.1. A projection of $c$ onto $W_{f}$ has the form

$$
P_{z}(x)=x-f(x) z
$$

for some $z \in f^{-1}(1)$. Moreover it holds

$$
\left\|P_{z}\right\|=\sup _{i \geq 1}\left\{\left|1-f_{i+1} z_{i}\right|+\left|z_{i}\right|\left(1-\left|f_{i+1}\right|\right)\right\} .
$$

Proof. The first part is well known (see, e.g, [3]). Now we will prove the formula (2.1). We have

$$
\begin{array}{r}
\left\|P_{z}\right\|=\sup _{x \in B_{c}} \sup _{i \geq 1}\left|\left(P_{z}(x)\right)_{i}\right|=\sup _{x \in B_{c}} \sup _{i \geq 1}\left|x_{i}-f(x) z_{i}\right|= \\
\sup _{x \in B_{c}} \sup _{i \geq 1}\left|\sum_{j=0}^{+\infty}\left(\delta_{i j}-f_{j+1} z_{i}\right) x_{j}\right| .
\end{array}
$$

Therefore, it holds

$$
\left\|P_{z}\right\| \leq \sup _{i \geq 1}\left(\sum_{j=0}^{+\infty}\left|\delta_{i j}-f_{j+1} z_{i}\right|\right) .
$$

Now let us consider, for every $i \geq 1$, the sequences $\left\{x^{(n, i)}\right\}_{n \geq 1} \subset B_{c}$ where $x^{(n, i)}=$ $\left(x_{1}^{(n, i)}, x_{2}^{(n, i)}, \ldots\right)$ is defined by:

$$
\left\{\begin{array}{cc}
x_{j}^{(n, i)}=\operatorname{sgn}\left(\delta_{i j}-f_{j+1} z_{i}\right) & \text { for } j \leq n \\
x_{j}^{(n, i)}=\operatorname{sgn}\left(-f_{1} z_{i}\right) & \text { for } j>n
\end{array} .\right.
$$

Then we have that, for every integers $n \geq 1$,

$$
\begin{gathered}
\sup _{i \geq 1}\left\|P_{z}\left(x^{(n, i)}\right)\right\|=\sup _{i \geq 1}\left|\sum_{j=0}^{+\infty}\left(\delta_{i j}-f_{j+1} z_{i}\right) x_{j}^{(n, i)}\right| \geq \sup _{1 \leq i \leq n}\left|\sum_{j=0}^{+\infty}\left(\delta_{i j}-f_{j+1} z_{i}\right) x_{j}^{(n, i)}\right|= \\
=\sup _{1 \leq i \leq n}\left|\sum_{j=0}^{n}\right| \delta_{i j}-f_{j+1} z_{i}\left|-z_{i} \operatorname{sgn}\left(-f_{1} z_{i}\right) \sum_{j=n+1}^{+\infty} f_{j+1}\right| .
\end{gathered}
$$

Therefore, we obtain that

$$
\left\|P_{z}\right\| \geq \sup _{i \geq 1}\left(\sum_{j=0}^{+\infty}\left|\delta_{i j}-f_{j+1} z_{i}\right|\right) .
$$

Hence, on combining (2.2) and (2.3), we conclude that

$$
\left\|P_{z}\right\|=\sup _{i \geq 1}\left(\sum_{j=0}^{+\infty}\left|\delta_{i j}-f_{j+1} z_{i}\right|\right) .
$$

Finally, an easy computation shows that

$$
\left\|P_{z}\right\|=\sup _{i \geq 1}\left\{\left|1-f_{i+1} z_{i}\right|+\left|z_{i}\right| \sum_{\substack{j=0 \\ j \neq i}}^{+\infty}\left|f_{j+1}\right|\right\}=
$$


HYPERPLANES IN THE SPACE OF CONVERGENT SEQUENCES AND PREDUALS OF $\ell_{1} 4$

$$
=\sup _{i \geq 1}\left\{\left|1-f_{i+1} z_{i}\right|+\left|z_{i}\right|\left(1-\left|f_{i+1}\right|\right)\right\}
$$

By means of the previous lemma, we are able to characterize the 1-complemented hyperplanes of $c$.

Proposition 2.2. A norm-1 projection of $c$ onto $W_{f}$ exists if and only if $\left|f_{j}\right| \geq \frac{1}{2}$ for some $j \geq 2$. Moreover there exists a unique norm-1 projection of c onto $W_{f}$ if and only if there exists a unique index $j_{0} \geq 2$ such that $\left|f_{j_{0}}\right| \geq \frac{1}{2}$.

Proof. By Lemma 2.1 $W_{f}$ is the rank of a norm-1 projection if and only if there exists $z \in c$ such that:

$$
\begin{gathered}
\left|1-f_{i+1} z_{i}\right|+\left|z_{i}\right|\left(1-\left|f_{i+1}\right|\right) \leq 1 \quad \forall i \geq 1 \\
\sum_{j=0}^{+\infty} f_{j+1} z_{j}=1 .
\end{gathered}
$$

Inequality (2.4) implies that $\operatorname{sgn}\left(f_{i+1}\right)=\operatorname{sgn}\left(z_{i}\right)$ for every $i \geq 1$. Then (2.4) becomes

and hence

$$
1-f_{i+1} z_{i}+\left|z_{i}\right|-z_{i} f_{i+1} \leq 1 \quad \forall i \geq 1
$$

$$
\left|z_{i}\right|\left(1-2\left|f_{i+1}\right|\right) \leq 0 \quad \forall i \geq 1 .
$$

Therefore for every $i$ such that $\left|f_{i+1}\right|<\frac{1}{2}$ it holds $z_{i}=0$. By equation (2.5) we conclude that there exists at least one index $j_{0} \geq 2 \operatorname{such}$ that $\left|f_{j_{0}}\right| \geq \frac{1}{2}$.

Now let us consider an element $z^{0} \in c$ such that

$$
z_{j_{0}-1}^{0}=\frac{1}{f_{j_{0}}}, \quad z_{j}^{0}=0 \quad \forall j \neq j_{0}-1 .
$$

It is easy to see that $z^{0}$ satisfies equations (2.4) and (2.5), $\left\|P_{z^{0}}\right\|=1$. Finally, if there is a unique index $j_{0}$ such that $\left|f_{j_{0}}\right| \geq \frac{1}{2}$, we remark that a unique projection $P_{z^{0}}$ exists (where $z^{0}$ is defined by (2.6) ). If there are two indexes $j_{1}$ and $j_{2}$ such that $\left|f_{j_{1}}\right|=\left|f_{j_{2}}\right|=\frac{1}{2}$ then both the projections $P_{z^{1}}$ and $P_{z^{2}}$ (where $z^{1}$ and $z^{2}$ are defined by (2.6) ) have norm 1 .

Lemma 2.1 allows us to give an explicit formula to compute the projection constant of the hyperplane $W_{f}$.

Proposition 2.3. Let $f \in \ell_{1}$ be such that $\|f\|_{\ell_{1}}=1$ and $\left|f_{j}\right|<\frac{1}{2}$ for every $j \geq 2$. Then

$$
\inf _{z \in f^{-1}(1)}\left\|P_{z}\right\|=1+\left(\left|f_{1}\right|+\sum_{j=1}^{+\infty} \frac{\left|f_{j+1}\right|}{1-2\left|f_{j+1}\right|}\right)^{-1}
$$

Proof. Let us consider the quantity

$$
\alpha_{N}=\left|f_{1}\right|+\sum_{j=1}^{N-1} \frac{\left|f_{j+1}\right|}{1-2\left|f_{j+1}\right|}+\operatorname{sgn}\left(f_{1}\right) \sum_{j=N}^{+\infty} f_{j+1} .
$$

We first remark that there exists $N_{0} \in \mathbb{N}$ such that for every $N \geq N_{0}$ it holds $\alpha_{N}>0$ and

$$
\alpha_{N} \geq \frac{\left|f_{k+1}\right|}{1-2\left|f_{k+1}\right|}
$$

for every $1 \leq k \leq N-1$. 
HYPERPLANES IN THE SPACE OF CONVERGENT SEQUENCES AND PREDUALS OF $\ell_{1} 5$

Let us consider the sequence $\left\{z^{N}\right\}_{N \geq N_{0}} \subset c$ defined by

$$
z^{N}=\lambda_{N}(\underbrace{\frac{\operatorname{sgn}\left(f_{2}\right)}{1-2\left|f_{2}\right|}, \ldots, \frac{\operatorname{sgn}\left(f_{N}\right)}{1-2\left|f_{N}\right|}}_{N-1}, \operatorname{sgn}\left(f_{1}\right), \operatorname{sgn}\left(f_{1}\right), \ldots),
$$

where $\lambda_{N}$ is a positive real number such that $f\left(z^{N}\right)=1$. Therefore, it is $\lambda_{N}=\alpha_{N}^{-1}$. Now, it holds

$$
\left\|P_{z^{N}}\right\|=\sup _{i \geq 1}\left\{\left|1-f_{i+1} z_{i}^{N}\right|+\left|z_{i}^{N}\right|\left(1-\left|f_{i+1}\right|\right)\right\} \leq 1+\lambda_{N}
$$

for every $N>N_{0}$. Indeed, by inequality (2.7) we obtain that $1-\lambda_{N} \frac{\left|f_{i+1}\right|}{1-2\left|f_{i+1}\right|} \geq 0$, and hence, for $1 \leq i \leq N-1$, we have

$$
1-\lambda_{N} \frac{\left|f_{i+1}\right|}{1-2\left|f_{i+1}\right|}+\lambda_{N}\left(\frac{1-\left|f_{i+1}\right|}{1-2\left|f_{i+1}\right|}\right)=1+\lambda_{N} .
$$

Moreover, for $i \geq N$

$\left|1-\lambda_{N} f_{i+1} \operatorname{sgn}\left(f_{1}\right)\right|+\lambda_{N}\left(1-\left|f_{i+1}\right|\right) \leq 1+\lambda_{N}\left|f_{i+1}\right|+\lambda_{N}-\lambda_{N}\left|f_{i+1}\right| \leq 1+\lambda_{N}$.

By (2.8) we have that $\inf _{z \in f^{-1}(1)}\left\|P_{z}\right\| \leq 1+\lambda$, where

$$
\lambda=\lim _{N} \lambda_{N}=\left(\left|f_{1}\right|+\sum_{j=1}^{+\infty} \frac{\left|f_{j+1}\right|}{1-2\left|f_{j+1}\right|}\right)^{-1} .
$$

We will finish the proof by showing that $\inf _{z \in f^{-1}(1)}\left\|P_{z}\right\|=1+\lambda$. Let us consider two different cases.

First, let us suppose that $\left|f_{1}\right|=1$ and hence $\lambda=1$. In this case it is well known that $\inf _{z \in f^{-1}(1)}\left\|P_{z}\right\|=2$ (see, e.g., [5]).

Finally, let $\left|f_{1}\right|<1$. By contradiction, let us suppose that there exists $\hat{z} \in f^{-1}(1)$ such that

$$
\left\|P_{\hat{z}}\right\|=\sup _{i \geq 1}\left\{\left|1-f_{i+1} \hat{z}_{i}\right|+\left|\hat{z}_{i}\right|\left(1-\left|f_{i+1}\right|\right)\right\}<1+\lambda
$$

hence

$$
\left|1-f_{i+1} \hat{z}_{i}\right|+\left|\hat{z}_{i}\right|\left(1-\left|f_{i+1}\right|\right)<1+\lambda
$$

for every $i \geq 1$ and then

$$
1-\left|f_{i+1}\right|\left|\hat{z}_{i}\right|+\left|\hat{z}_{i}\right|-\left|\hat{z}_{i}\right|\left|f_{i+1}\right|<1+\lambda .
$$

Therefore, for every $i \geq 1$, it holds that

$$
\left(1-2\left|f_{i+1}\right|\right)\left|\hat{z}_{i}\right|<\lambda \text {. }
$$

Moreover, the last relation gives that

$$
\left|\hat{z}_{0}\right|=\lim _{i}\left|\hat{z}_{i}\right| \leq \lim _{i} \frac{\lambda}{1-2\left|f_{i+1}\right|}=\lambda
$$

Since there exists at least one index $\hat{j} \geq 1$ such that $f_{\hat{j}+1} \neq 0$, by using inequalities (2.10) and (2.11) and by recalling (2.9), we conclude that

$$
\sum_{j=0}^{+\infty} f_{j+1} \hat{z}_{j} \leq\left|f_{1}\right|\left|\hat{z}_{0}\right|+\sum_{j=1}^{+\infty}\left|f_{j+1}\right|\left|\hat{z}_{j}\right|<\lambda\left(\left|f_{1}\right|+\sum_{j=1}^{+\infty} \frac{\left|f_{j+1}\right|}{1-2\left|f_{j+1}\right|}\right)=1 .
$$

The last inequality is a contradiction because it holds $\sum_{j=0}^{+\infty} f_{j+1} \hat{z}_{j}=1$. 


\section{ISOMETRIES BETWEEN THE HYPERPLANES OF $c$ AND THE SPACES $c$ AND $c_{0}$}

In this section we show that the isometric structure of the hyperplanes is completely described whenever the associated projection constant assumes the extreme values. Indeed, we will prove that $W_{f}$ is isometric to $c$ if and only if it is 1complemented, whereas $W_{f}$ is isometric to $c_{0}$ if and only if its projection constant is 2 . We begin to study the 1-complemented hyperplanes of $c$. The first step shows that a 1-complemented hyperplane is isometric to $c$.

Proposition 3.1. If $W_{f} \subset c$ is 1-complemented then $W_{f}$ is isometric to $c$.

Proof. By Theorem 2.2, there exists $j_{0} \geq 2$ such that $\left|f_{j_{0}}\right| \geq \frac{1}{2}$. Now, let us consider $T: c \rightarrow W_{f}$ defined by

$$
T\left(x_{1}, x_{2}, \ldots\right)=(x_{1}, \ldots, x_{j_{0}-2}, \underbrace{\alpha}_{j_{0}-1}, x_{j_{0}-1}, x_{j_{0}}, \ldots)
$$

where

$$
\alpha=-\frac{1}{f_{j_{0}}}\left(\sum_{j=0}^{j_{0}-2} f_{j+1} x_{j}+\sum_{j=j_{0}}^{+\infty} f_{j+1} x_{j-1}\right) .
$$

The inverse of $T$ is $T^{-1}: W_{f} \rightarrow c$ acts on $y=\left(y_{1}, y_{2}, \ldots\right) \in W_{f}$ by deleting the $\left(j_{0}-1\right)$-th component of $y$. Moreover, if $x \in c$, then

$$
\begin{gathered}
|\alpha| \leq \frac{1}{\left|f_{j_{0}}\right|}\left(\sum_{j=0}^{j_{0}-2}\left|f_{j+1}\right|\left|x_{j}\right|+\sum_{j=j_{0}}^{+\infty}\left|f_{j+1}\right|\left|x_{j-1}\right|\right) \\
\leq \frac{1}{\left|f_{j_{0}}\right|}\left(\sum_{j=0}^{j_{0}-2}\left|f_{j+1}\right|+\sum_{j=j_{0}}^{+\infty}\left|f_{j+1}\right|\right)\|x\|=\frac{1}{\left|f_{j_{0}}\right|}\left(\sum_{\substack{j=0 \\
j \neq j_{0}-1}}^{+\infty}\left|f_{j+1}\right|\right)\|x\|= \\
=\frac{1}{\left|f_{j_{0}}\right|}\left(1-\left|f_{j_{0}}\right|\right)\|x\| \leq\|x\| .
\end{gathered}
$$

Therefore $T$ is an isometry between $W_{f}$ and $c$.

In order to prove the reverse implication we need to investigate the family of the isometries on $c$ with 1-codimensional range. To this aim, we adapt to our framework some results from [6] (Theorem 2.1 and Lemma 2.2) about the isometries on the space of continuous functions defined on a compact set. It is worth to remark that the mentioned results in [6] do not refer to general isometries but they consider only shift operators. Nevertheless, by considering the proofs of these results, it is easy to see that they hold for general isometries with 1-codimensional range.

As it is well known the space $c$ can be seen as the space $\mathcal{C}\left(\mathbb{N}^{*}\right)$ of continuous function on $\mathbb{N}^{*}$, where $\mathbb{N}^{*}$ denotes the Alexandroff one-point compactification of the set of positive integers. For the sake of convenience, we denote by 0 the unique limit point of $\mathbb{N}^{*}$.

Theorem 3.2. (Theorem 2.1 and Lemma 2.2 in [6]). Let $T: \mathcal{C}\left(\mathbb{N}^{*}\right) \rightarrow \mathcal{C}\left(\mathbb{N}^{*}\right)$ be an isometry with 1-codimensional range. Then there exist a closed subset $M$ of $\mathbb{N}^{*}$, a continuous and surjective function $\varphi: M \rightarrow \mathbb{N}^{*}$ where $\varphi^{-1}(n)$ has at most two elements for each $n \in \mathbb{N}^{*}$ and a sequence $\left\{\varepsilon_{n}\right\}_{n \in \mathbb{N}^{*}}$ where $\left|\varepsilon_{n}\right|=1$ such that

$$
(T x)_{n}=\varepsilon_{n} x_{\varphi(n)} \quad \text { for every } n \in M .
$$

Moreover, only one of the two following alternatives holds: 
HYPERPLANES IN THE SPACE OF CONVERGENT SEQUENCES AND PREDUALS OF $\ell_{1} 7$

(1) $M=\mathbb{N}^{*} \backslash\{\bar{n}\}$ where $\bar{n}$ is a positive integer. In addition, in this case $\varphi$ is also injective;

(2) $M=\mathbb{N}^{*}$ and, if there exists $n^{\prime} \in \mathbb{N}^{*}$ such that the set $\varphi^{-1}\left(n^{\prime}\right)$ has two elements, then $\varphi^{-1}(n)$ is a singleton for every $n \in \mathbb{N}^{*} \backslash\left\{n^{\prime}\right\}$.

Now, by means of the previous theorem, we will show that $W_{f}$ is 1-complemented whenever it is isometric to $c$.

Proposition 3.3. If $W_{f} \subset c$ is isometric to $c$ then $W_{f}$ is 1-complemented.

Proof. By recalling Proposition [2.2, in order to prove the theorem is sufficient to show that there exists $j_{0} \geq 2$ such that $\left|f_{j_{0}}\right| \geq \frac{1}{2}$ whenever there exists an isometry $T: c \rightarrow W_{f}$.

First of all, by the continuity of $\varphi$ it is easy to see that

$$
\varphi(0)=0 .
$$

Now, let us consider case (1) of Theorem 3.2 and let us suppose that $M=\mathbb{N}^{*} \backslash\{1\}$ without loss of generality. Therefore equation (3.1) is true for every $n \geq 2$. Hence, all the components of $T x$ are known except $(T x)_{1}=z$. Since $T x \in W_{f}$ for every $x \in c$, then it holds

$$
f_{1} \varepsilon_{0} x_{0}+f_{2} z+\sum_{j=2}^{+\infty} f_{j+1} \varepsilon_{j} x_{\varphi(j)}=0 .
$$

By the injectivity of $\varphi$, we can choose $x_{N} \in c$ such that

$$
\varepsilon_{n}\left(x_{N}\right)_{\varphi(n)}=\operatorname{sgn} f_{n+1}
$$

for every $2 \leq n \leq N$. All the other components of $x_{N}$ are equal to a value $x_{0}$ given by

By (3.2), we have

$$
\varepsilon_{0} x_{0}=\operatorname{sgn} f_{1}
$$

$$
\left|f_{1}\right|+f_{2} z_{N}+\left|f_{3}\right|+\ldots+\left|f_{N+1}\right|+x_{0} \sum_{j=N+1}^{+\infty} f_{j+1} \varepsilon_{j}=0
$$

where $z_{N}=\left(T x_{N}\right)_{1}$. Since $T$ is an isometry $\left|z_{N}\right| \leq 1$ and hence, up to a subsequence, we can suppose that $z_{N}$ converges to $\hat{z}$. Therefore, as $N \rightarrow \infty$, it holds

$$
\left|f_{1}\right|+f_{2} \hat{z}+\sum_{j=2}^{+\infty}\left|f_{j+1}\right|=f_{2} \hat{z}+1-\left|f_{2}\right|=0
$$

and hence $\hat{z}=-\frac{1-\left|f_{2}\right|}{f_{2}}$. Since $|\hat{z}| \leq 1$, we conclude that $\left|f_{2}\right| \geq \frac{1}{2}$.

Now, let us study the case (2) of Theorem 3.2 where $M=\mathbb{N}^{*}$. We consider three different situations.

- Let $\varphi^{-1}(n)$ be a pair for an element $n \in \mathbb{N}^{*}, n \neq 0$. Without loss of generality, we can suppose that the map $\varphi: \mathbb{N}^{*} \rightarrow \mathbb{N}^{*}$ is one-to-one everywhere except at the point 1 where it holds

$$
\varphi^{-1}(1)=\{1,2\} \text {. }
$$

Since $T x \in W_{f}$ then we have

$$
f_{1} \varepsilon_{0} x_{0}+f_{2} \varepsilon_{1} x_{1}+f_{3} \varepsilon_{2} x_{1}+\sum_{j=3}^{+\infty} f_{j+1} \varepsilon_{j} x_{\varphi(j)}=0 .
$$

By the injectivity of $\varphi$, we can choose $x_{N} \in c$ such that

$$
\varepsilon_{n}\left(x_{N}\right)_{\varphi(n)}=\operatorname{sgn} f_{n+1}
$$


HYPERPLANES IN THE SPACE OF CONVERGENT SEQUENCES AND PREDUALS OF $\ell_{1} 8$

for every $3 \leq n \leq N$. Moreover, the component $\left(x_{N}\right)_{1}$ is chosen to satisfies $\varepsilon_{1}\left(x_{N}\right)_{1}=\operatorname{sgn} f_{2}$. All the other components of $x_{N}$ are equal to the value $x_{0}$ given by

$$
\varepsilon_{0} x_{0}=\operatorname{sgn} f_{1}
$$

Since $T x_{N} \in W_{f}$, by (3.3), we have

$$
\left|f_{1}\right|+\left|f_{2}\right|+f_{3} \varepsilon_{1} \varepsilon_{2} \operatorname{sgn} f_{2}+\ldots+\left|f_{N+1}\right|+x_{0} \sum_{j=N+1}^{+\infty} f_{j+1} \varepsilon_{j}=0 .
$$

Therefore, as $N \rightarrow \infty$, it holds

$$
\left|f_{1}\right|+\left|f_{2}\right|+f_{3} \varepsilon_{1} \varepsilon_{2} \operatorname{sgn} f_{2}+\sum_{j=3}^{+\infty}\left|f_{j+1}\right|=0
$$

and hence

$$
1-\left|f_{3}\right|+f_{3} \varepsilon_{1} \varepsilon_{2} \operatorname{sgn} f_{2}=0 .
$$

Similarly, by choosing $\left(x_{N}\right)_{1}$ to satisfy $\varepsilon_{2}\left(x_{N}\right)_{1}=\operatorname{sgn} f_{3}$, we obtain an analogous equation for $f_{2}$

$$
1-\left|f_{2}\right|+f_{2} \varepsilon_{1} \varepsilon_{2} \operatorname{sgn} f_{3}=0 .
$$

Equations (3.4) and (3.5) hold true simultaneously only if $\left|f_{2}\right|=\left|f_{3}\right|=\frac{1}{2}$.

- Let $\varphi^{-1}(0)$ be a pair. Without loss of generality we can suppose that

$$
\varphi^{-1}(0)=\{0,1\} \text {. }
$$

Analysis similar to that in the previous point shows that $\left|f_{1}\right|=\left|f_{2}\right|=\frac{1}{2}$.

- Finally, let $\varphi$ be injective. The same argument applied above yields the following contradiction

$$
\sum_{j=1}^{+\infty}\left|f_{j}\right|=0
$$

Now we study the hyperplanes of $c$ that are isometric to $c_{0}$. First, we prove that hyperplanes of $c$ with projection constant equal to 2 is an isometric copy of $c_{0}$.

Proposition 3.4. If $W_{f}$ is such that $\inf _{z}\left\|P_{z}\right\|=2$ (where $P_{z}: c \rightarrow W_{f}$ is a projection) then $W_{f}$ is isometric to $c_{0}$.

Proof. Let us recall that, by Proposition 2.3 .

$$
\inf _{z}\left\|P_{z}\right\|=1+\left(\left|f_{1}\right|+\sum_{j=1}^{+\infty} \frac{\left|f_{j+1}\right|}{1-2\left|f_{j+1}\right|}\right)^{-1} .
$$

Then, since $\inf _{z}\left\|P_{z}\right\|=2$, we have

$$
\left|f_{1}\right|+\sum_{j=1}^{+\infty} \frac{\left|f_{j+1}\right|}{1-2\left|f_{j+1}\right|}=1
$$

Since $\|f\|_{1}=1$, it holds

$$
\sum_{j=1}^{+\infty} \frac{\left|f_{j+1}\right|}{1-2\left|f_{j+1}\right|}=\sum_{j=1}^{+\infty}\left|f_{j+1}\right|
$$

and hence

$$
\sum_{j=1}^{+\infty}\left(\frac{\left|f_{j+1}\right|}{1-2\left|f_{j+1}\right|}-\left|f_{j+1}\right|\right)=\sum_{j=1}^{+\infty}\left(\frac{2\left|f_{j+1}\right|^{2}}{1-2\left|f_{j+1}\right|}\right)=0
$$


Since $\left|f_{j+1}\right|<\frac{1}{2}$ for every $j=1,2,3, \ldots$ (otherwise $\inf _{z}\left\|P_{z}\right\|=1$ by Proposition 2.2), the last equality holds if and only if $f_{j+1}=0$ for every $j=1,2, \ldots$. Therefore $f= \pm(1,0,0, \ldots)$ and hence $W_{f} \simeq c_{0}$.

Now we prove that there exists a unique hyperplane of $c$ isometric to $c_{0}$. This assertion follows directly from a simple lemma that can be stated in a more general setting.

Lemma 3.5. Let $V$ be a subspace of $\mathcal{C}(K)$ where $K$ is a compact metric space. If $V$ is isometric to $c_{0}$ then there exists $p \in K$ such that

$$
V \subseteq\{f \in \mathcal{C}(K): f(p)=0\}
$$

Proof. Let $T: V \rightarrow c_{0}$ be an isometry. Therefore $T^{*}: \ell_{1} \rightarrow V^{*}$ is also an isometry and hence $T^{*}\left(e_{n}\right) \in \operatorname{ext} B_{V^{*}}$ where $e_{n}$ is the $n$-th element of the standard basis of $\ell_{1}$. By Lemma 6, p.441 in [4], we have

$$
T^{*}\left(e_{n}\right)= \pm x_{p_{n}}^{*}
$$

where $x_{p}^{*}$ is the evaluation functional. Since $\left\{e_{n}\right\}$ is a weak ${ }^{*}$-null sequence, $\left|x_{p_{n}}^{*}(f)\right| \rightarrow$ 0 for every $f \in V$. Now, by the compactness of $K$, there exists an element $p \in K$ such that $f(p)=0$ for every $f \in V$.

If we take $\mathcal{C}(K)=\mathcal{C}\left(\mathbb{N}^{*}\right)=c$ and $V=W_{f}$ in the previous lemma, then necessarily it holds

$$
W_{f}=\left\{x \in c: x_{0}=\lim _{i} x_{i}=0\right\}
$$

since $W_{f}$ has codimension 1 .

\section{Duality Between $W_{f}$ And $\ell_{1}$ And some APplications}

We start this section by characterizing the hyperplanes of $c$ such that their duals are isometric to $\ell_{1}$. Despite its simple proof, this result plays a central role in our approach since it allows us conclude the proof of our main result, namely Theorem 1.2

Proposition 4.1. There exists $j_{0} \in \mathbb{N}$ such that $\left|f_{j_{0}}\right| \geq \frac{1}{2}$ if and only if $W_{f}^{*} \simeq \ell_{1}$.

Proof. Let us denote by $V_{f}$ the subspace of $c_{0}$ defined as $V_{f}=\operatorname{ker} f \subset c_{0}$. Since

$$
V_{f}^{*} \simeq \ell_{1} /[f]
$$

then $W_{f}^{*} \simeq V_{f}^{*}$. By Theorem 1.1 we have that $W_{f}^{*} \simeq \ell_{1}$ if and only if there exists $j_{0} \in \mathbb{N}$ such that $\left|f_{j_{0}}\right| \geq \frac{1}{2}$.

A consequence of the main result of our paper (Theorem 1.2) is the classification of the hyperplanes of $c$ such that their duals are isometric copies of $\ell_{1}$.

Remark 4.2. The hyperplanes $W_{f}$ of $c$ such that $W_{f}^{*} \simeq \ell_{1}$ can be divided into three distinct classes:

- $W_{f} \simeq c$ (or, equivalently, there exists $j_{0} \geq 2$ such that $\left|f_{j_{0}}\right| \geq \frac{1}{2}$ );

- $W_{f}$ is isometric neither to $c$ nor to $c_{0}$ (or, equivalently, $\frac{1}{2} \leq\left|f_{1}\right|<1$ and $\left|f_{j}\right|<\frac{1}{2}$ for every $\left.j \geq 2\right)$

- $W_{f} \simeq c_{0}$ (or, equivalently, $\left.\left|f_{1}\right|=1\right)$.

The most interesting situations occur when $W_{f}$ is isometric neither to $c$ nor to $c_{0}$. In these cases we study the $\sigma\left(\ell_{1}, W_{f}\right)$-limit of the standard basis of $\ell_{1}$. It is worth to mention that in order to reach this aim we explicitly describe the duality between $W_{f}$ and $\ell_{1}$. 
HYPERPLANES IN THE SPACE OF CONVERGENT SEQUENCES AND PREDUALS OF $\ell_{1} 10$

Theorem 4.3. Let $W_{f} \subset c$ be such that $W_{f}^{*} \simeq \ell_{1}, \frac{1}{2} \leq\left|f_{1}\right|<1$ and $\left|f_{j}\right|<\frac{1}{2}$ for every $j \geq 2$. If $\left\{e_{n}\right\}$ is the standard basis of $\ell_{1}$, then

$$
e_{n} \stackrel{\sigma\left(\ell_{1}, W_{f}\right)}{\longrightarrow} \hat{e}
$$

where $\hat{e}=\left(-\frac{f_{2}}{f_{1}},-\frac{f_{3}}{f_{1}},-\frac{f_{4}}{f_{1}}, \ldots\right)$.

Proof. Consider the map $\phi: \ell_{1} \rightarrow W_{f}^{*}$ defined by

$$
(\phi(y))(x)=\sum_{j=1}^{+\infty} x_{j} y_{j}
$$

where $y=\left(y_{1}, y_{2}, \ldots\right) \in \ell_{1}$ and $x=\left(x_{1}, x_{2}, \ldots\right) \in W_{f}$.

It is easy to see that $\phi\left(\ell_{1}\right)=W_{f}^{*}$, and, for any $y \in \ell_{1}$,

$$
\|\phi(y)\|_{W_{f}^{*}} \leq\|y\|_{\ell_{1}} \text {. }
$$

Now, for a given $y=\left(y_{1}, y_{2}, \ldots\right) \in \ell_{1}$, consider the points $x^{N}, N=1,2, \ldots$, defined as

$$
x^{N}=\left(\operatorname{sgn}\left(y_{1}\right), \operatorname{sgn}\left(y_{2}\right), \ldots, \operatorname{sgn}\left(y_{N}\right), x_{0}^{N}, x_{0}^{N}, \ldots\right),
$$

where $x_{0}^{N}$ satisfies the following equation

$$
f_{1} x_{0}^{N}+\sum_{j=1}^{N} f_{j+1} \operatorname{sgn}\left(y_{j}\right)+x_{0}^{N} \sum_{j=N+1}^{+\infty} f_{j+1}=0 .
$$

It is clear that $x^{N} \in W_{f}$ for all $N$. Moreover, for any $N \geq N_{0}$, where $N_{0}$ is such that $\sum_{j=N_{0}+1}^{+\infty}\left|f_{j+1}\right|<\frac{1}{2}$, we have $x^{N} \in B_{W_{f}}$. Indeed, for every $N \geq N_{0}$, we have

$$
\begin{gathered}
\left|x_{0}^{N}\right|=\left|\frac{-\sum_{j=1}^{N} f_{j+1} \operatorname{sgn}\left(y_{j}\right)}{f_{1}+\sum_{j=N+1}^{+\infty} f_{j+1}}\right| \leq \frac{\sum_{j=1}^{N}\left|f_{j+1}\right|}{\left|f_{1}+\sum_{j=N+1}^{+\infty} f_{j+1}\right|} \\
=\frac{1-\left|f_{1}\right|-\sum_{j=N+1}^{+\infty}\left|f_{j+1}\right|}{\left|f_{1}+\sum_{j=N+1}^{+\infty} f_{j+1}\right|} \leq \\
\leq \frac{\frac{1}{2}-\sum_{j=N+1}^{+\infty}\left|f_{j+1}\right|}{\frac{1}{2}-\sum_{j=N+1}^{+\infty}\left|f_{j+1}\right|}=1 .
\end{gathered}
$$

Now, for every $N \geq N_{0}$, we have

$$
\|\phi(y)\|_{W_{f}^{*}} \geq\left|(\phi(y))\left(x^{N}\right)\right|=\left|\sum_{j=1}^{N}\right| y_{j}\left|+x_{0}^{N} \sum_{j=N+1}^{+\infty} y_{j}\right| .
$$

Letting $N \rightarrow+\infty$ we get

$$
\|\phi(y)\|_{W_{f}^{*}} \geq\|y\|_{\ell_{1}} .
$$

By (4.1) and (4.2) we conclude that the map $\phi$ is an isometry.

Finally, for any $x=\left(x_{1}, x_{2}, \ldots\right) \in W_{f}$, we obtain

$$
\lim _{n \rightarrow \infty}\left(\phi\left(e_{n}\right)\right)(x)=\lim _{n \rightarrow \infty} x_{n}=x_{0}=-\frac{1}{f_{1}} \sum_{j=1}^{\infty} f_{j+1} x_{j}=(\phi(\hat{e}))(x) .
$$


The last result has some interesting consequences.

First of all, by recalling Lemma 2 in [1], that asserts "that the $w^{*}$-closure of the $\ell_{1}$-(standard) basis is the only thing that is important" to describe the structure of an $\ell_{1}$-predual space, we obtain a complete isometric descriptions of the preduals of $\ell_{1}$ under the additional assumption that its standard basis is $\sigma\left(\ell_{1}, X\right)$-convergent.

Corollary 4.4. Let $X$ be a Banach space such that $X^{*}=\ell_{1}$. If the standard basis $\left\{e_{n}\right\}$ of $\ell_{1}$ is a $\sigma\left(\ell_{1}, X\right)$-convergent sequence, then there exists $f \in \ell_{1}$ with $\|f\|_{\ell_{1}}=1$ such that $X$ is isometric to $W_{f}$.

Proof. Let $\hat{e}=\left(\hat{e}_{1}, \hat{e}_{2}, \ldots\right) \in \ell_{1}$ be the $\sigma\left(\ell_{1}, X\right)$-limit of $\left\{e_{n}\right\}$. If $\left|\hat{e}_{j}\right|<1$ for every $j \geq 1$, then by Theorem 4.3 and Lemma 2 in [1] we have that $X \simeq W_{f}$ where $f=\left(f_{1}, f_{2}, \ldots\right) \in \ell_{1}$ is defined by

$$
f_{1}=\frac{1}{1+\sum_{n=1}^{\infty}\left|\hat{e}_{n}\right|}, \quad f_{n}=-\frac{\hat{e}_{n-1}}{1+\sum_{n=1}^{\infty}\left|\hat{e_{n}}\right|} \text { for every } n \geq 2 .
$$

Now, it remains to consider the case where $\hat{e}=e_{m}$ for a fixed $m \geq 1$. Under this assumption it is easy to show that $X \simeq c$ and hence $X \simeq W_{f}$ for every $f \in \ell_{1}$ such that there exists $j_{0} \geq 2$ such that $\left|f_{j_{0}}\right| \geq \frac{1}{2}$.

Finally, we obtain some additional information about the isometric structure of the hyperplanes $W_{f}$. Let us introduce the following notations: by $\alpha$ we denote a countable ordinal and $C(\alpha)$ is the space of all continuous real-valued functions on the ordinals less than or equal to $\alpha$ with the order topology.

Corollary 4.5. There exists a countable ordinal $\alpha$ such that $W_{f}$ is isometric to a quotient of $C(\alpha)$ if and only if one of the following conditions holds:

(1) there exists an index $j_{0} \geq 2$ such that $\left|f_{j_{0}}\right| \geq \frac{1}{2}$;

(2) $\frac{1}{2} \leq\left|f_{1}\right| \leq 1$ and $\left|f_{j}\right|<\frac{1}{2}$ for all $j \geq 2$ and $f=\left(f_{1}, f_{2}, \ldots, f_{n}, 0,0, \ldots, 0, \ldots\right)$ for some $n \in \mathbb{N}$.

Proof. First of all, by Theorem 1.2 we have that condition (1) is satisfied if and only if $W_{f} \simeq c$. Now, it remains to consider the case where $\frac{1}{2} \leq\left|f_{1}\right| \leq 1$ and $\left|f_{j}\right|<\frac{1}{2}$ for all $j \geq 2$. Let us suppose $f=\left(f_{1}, f_{2}, \ldots, f_{n}, 0,0, \ldots\right)$ for some $n \geq 2$. Then $W_{f} \subset c$ is isometric to a quotient of $C(\omega \cdot n)$. To see it, consider the sequence of measures $\mu_{i}$ defined by $\mu_{i}=\delta_{\omega \cdot i}$ for $i=1,2, \ldots, n-1$, and, for $i=n, n+1, n+2, \ldots$, (we put $\left.\delta_{\omega \cdot 0+i}:=\delta_{i}\right)$

$$
\mu_{i}=-\frac{1}{f_{1}} \sum_{j=2}^{n} f_{j} \cdot \delta_{\omega \cdot(j-2)+i}+\frac{2\left|f_{1}\right|-1}{\left|f_{1}\right| \cdot i} \sum_{j=1}^{i}(-1)^{j} \cdot \delta_{\omega \cdot(n-1)+\frac{i \cdot(i-1)}{2}+j} .
$$

Now it is enough to apply Theorem 4.3 of the present paper and Proposition 3 in [1], with the mapping $\phi$ given by $\phi\left(e_{i}\right)=\mu_{i}$. To show that condition (2) implies that $W_{f}$ is isometric to a quotient of $C(\alpha)$ for some $\alpha$, it is sufficient to consider Proposition 6 in [1] and Theorem 4.3 of the present paper.

\section{REFERENCES}

[1] D. E. Alspach. A $\ell_{1}$-predual which is not isometric to a quotient of $C(\alpha)$. arXiv:math/9204215 1 [math.FA] 27 Apr 1992.

[2] M. Baronti. Norm-one projections onto subspaces of $\ell^{\infty}$. Arch. Math. 51 (1988), 242-246.

[3] J. Blatter, E. W. Cheney. Minimal Projections on Hyperplanes in Sequence Spaces. Annali Mat. Pura Appl. 101 (1974), 215-227.

[4] N. Dunford, J. T. Schwartz. Linear operators. Part I. General theory. Reprint of the 1958 original. John Wiley \& Sons, Inc., New York, 1988.

[5] M. Fabian, P. Habala, P. Hájek, V. Montesinos Santalucía, J. Pelant, V. Zizler. Functional analysis and infinite-dimensional geometry. CMS Books in Mathematics, 8. Springer-Verlag, New York, 2001. 
HYPERPLANES IN THE SPACE OF CONVERGENT SEQUENCES AND PREDUALS OF $\ell_{1} 12$

[6] A. Gutek, D. Hart, J. Jamison, M. Rajagopalan. Shift operators on Banach spaces. J. Funct. Anal. 101 (1991), 97-119.

[7] J. Lindenstrauss, L. Tzafriri. Classical Banach spaces. I. Sequence spaces. Ergebnisse der Mathematik und ihrer Grenzgebiete, Vol. 92. Springer-Verlag, Berlin-New York, 1977.

Dipartimento di Scienza e Alta Tecnologia, Università dell'Insubria, via Valleggio 11, 22100 Como, Italy

E-mail address: emanuele.casini@uninsunbria.it

Dipartimento di Discipline Matematiche, Finanza Matematica ed Econometria, Università Cattolica del Sacro Cuore, Via Necchi 9, 20123 Milano, Italy

E-mail address: enrico.miglierina@unicatt.it

Institute of Mathematics, Maria Curie-Skeodowska University, Pl. Marii CurieSklodowskiej 5 20-031 Lublin, Poland

E-mail address: piasecki@hektor.umcs.lublin.pl 